\title{
Nuevo hallazgo de Nardophyllum genistoides (Phil.) Gray (Asteraceae) en Chile central.
}

\author{
New finding of Nardophyllum genistoides (Phil.) Gray (Asteraceae) in Central Chile
}

\author{
Mélica Muñoz-Schick ${ }^{1}$, Vanezza Morales ${ }^{2}$, María Eugenia Cruzat $^{3}$ \& Andrés Moreira-Muñoz ${ }^{2}$ \\ ${ }^{1}$ Museo Nacional de Historia Natural, Casilla 787, Santiago, Chile, \\ ${ }^{2}$ Instituto de Geografía, Pontificia Universidad Católica de Chile, Av. Vicuña Mackenna 4860. Santiago, Chile. \\ ${ }^{3}$ Colegio Francisco de Asís, Cerro Catedral Norte 12.150, Las Condes, Santiago, Chile. \\ mmunoz@mnhn.cl
}

\begin{abstract}
Nardophyllum genistoides (Phil.) Gray is recollected after a century and a half, in the same Region Metropolitana, Chile, at a new locality south of the original site. It grows around 1.600 masl, in the Río Clarillo National Reserve, associated to the sclerophyllous forest. The species is very rare, because only one plant was found.
\end{abstract}

Dolichogyne genistoides fue descrita por Rodulfo Amando Philippi (Linnaea 28: 738) basándose en un ejemplar recolectado por Germain en febrero de 1857, en la cordillera de Santiago (Philippi 1858). Dicho ejemplar corresponde al SGO 62028 y debe ser considerado como el lectotipo de la especie (Fig. 1).

Hasta este momento dicho ejemplar se consideraba dudoso, pues la fecha de descripción de Philippi ha sido referida al año 1856. Sin embargo de acuerdo a Stafleu \& Cowan (1985:198) el volumen 28 de Linnaea se publicó en 6 partes, comprendiendo la sexta de ellas las páginas 641 al 767 y fue publicada en febrero de 1858. En las revisiones del género, tanto Cabrera (1954) como Bonifacino (2009) indican el año de publicación como 1856, pero consideran el ejemplar del año 1857 como el tipo.

Un segundo ejemplar que se conserva en el Herbario SGO fue recolectado por L. Landbeck, en cordillera de Las Arañas, enero 1861 (SGO 71787). Del primero de ellos existen duplicados en BM y LP, y de ambos existe una carpeta en Kew (foto conservada en SGO), todos se consideran isotipos. Al analizar la foto en Kew, de ejemplares enviados por el propio Philippi, hemos podido deducir que se trata de duplicados de los 2 ejemplares, ya que la planta izquierda arriba se asemeja a la de Germain y la inferior se parece a la de Landbeck. La especie fue transferida por Gray (1873) al género Nardophyllum como $N$. genistoides (Phil.) A.Gray.
Nardophyllum es un género andino-patagónico con 5 especies: $N$. bryoides (Lam.) Cabrera, de Argentina y Chile: regiones Aisén y Magallanes, $N$. chiliotrichioides (J.Remy) A. Gray, de Argentina y Chile: regiones Coquimbo y Metropolitana, N. genistoides, endémica de Chile: Región Metropolitana, $N$. lanatum (Meyen) Cabrera, endémica de Chile: regiones de Atacama a O'Higgins y N. patagonicum (Cabrera) G.L.Nesom, endémica de Argentina (Bonifacino 2009). Nardophyllum armatum (Wedd.) Reiche, fue transferida recientemente a Ocyroe armata (Wedd.) Bonif. (2008) con distribución en Bolivia, Argentina y Chile.

Las dos colecciones mencionadas eran las únicas conocidas a la fecha, lo cual no obstante permitió a Bonifacino (2009) considerarla como una buena especie. En el marco de nuestra investigación de la biogeografía de las Asteráceas de Chile, un grupo de colaboradores encontró un ejemplar en la Reserva Nacional Río Clarillo en enero 2010. Para corroborar la localidad exacta así como su abundancia, se realizó una nueva excursión el 3 de febrero de 2010. Se llegó al mismo ejemplar, y a pesar de una intensa búsqueda en los alrededores, no se encontraron más ejemplares.

El ejemplar corresponde a un subarbusto quebradizo de $22 \mathrm{~cm}$, que se apoyaba sobre una roca al lado del sendero, bajo el bosque de peumo Cryptocarya alba (Molina) Looser. Crecían alrededor Solenomelus pedunculatus (Gillies ex Hook.) Hochr. y Mutisia subulata Ruiz et Pav. La comunidad corresponde al Bosque Esclerófilo, cerca de la transición a Bosque Subandino (Teillier et al. 2005). 


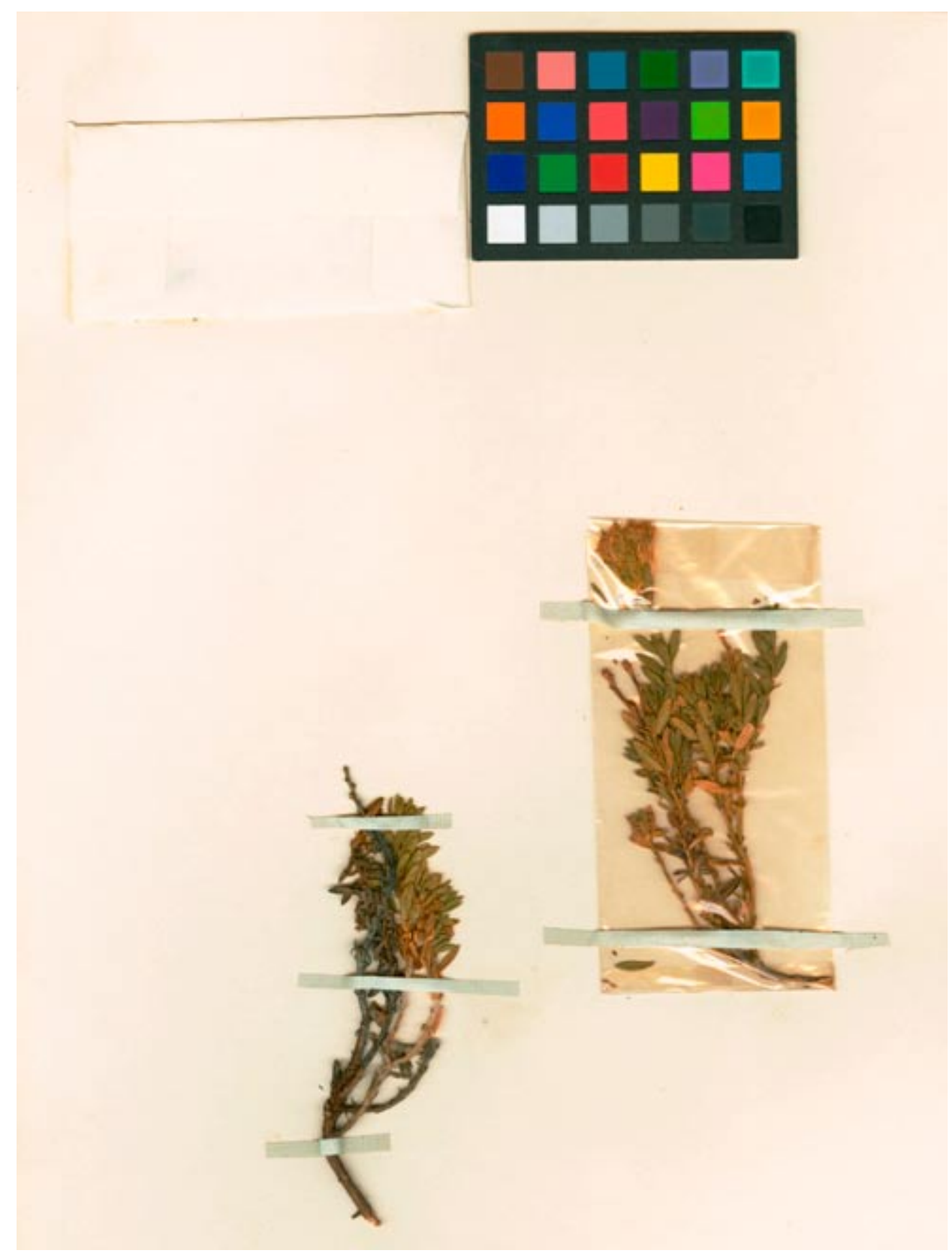

FIGURA 1. Dolichogyne genistoides, Lectotipo (SGO).

FIGURE 1. Dolichogyne genistoides, Lectotype (SGO). 
DESCRIPCIÓN

Subarbusto de 18-22 cm de alto, algo decumbente en la base, con ramas frágiles, fácilmente quebradizas y densamente cubiertas de pelos glandulares, hojas angostamente elípticas, de 10-22 $\mathrm{mm}$ de largo por 2-8 $\mathrm{mm}$ de ancho, subsésiles, densamente cubiertas por pelos glandulares en ambas superficies, con el nervio medio notorio por el envés, bordes revolutos. Capítulos solitarios, cortamente pedunculados; brácteas del involucro igualmente cubiertas de glándulas, ovado-lanceoladas, agudas; flores de color amarillo oro, con estilos amarillos muy sobresalientes, ramas estigmáticas del mismo largo de la parte que sobresale del estilo por sobre la corola, dispuestas formando una elipse. La planta posee una agradable fragancia cítrica (Fig. 2 A,B,C).

Bonifacino (2009) realiza una amplia descripción y una lámina muy completa basado principalmente en los ejemplares depositados en BM y LP. Con el material estudiado a esta fecha podemos confirmar que se trata de una buena especie del género Nardophyllum.
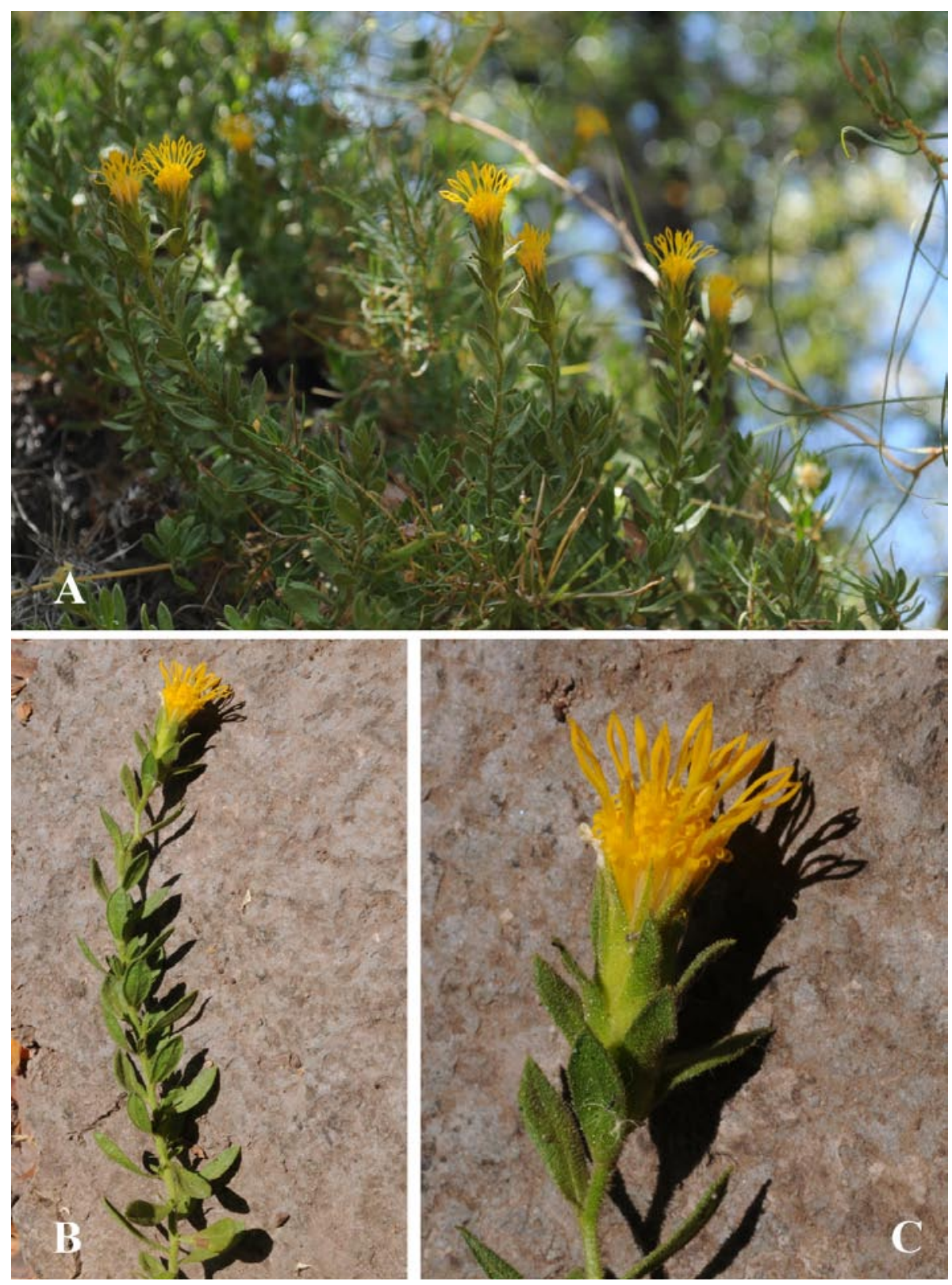

Figura 2. A. Hábito de Nardophyllum genistoides, B. Detalle de hojas, involucro y flores, C. Detalle de los largos estilos y estigmas (Fotografías de A. Moreira-Muñoz).

Figure 2. A. Habit of Nardophyllum genistoides, B. Leaves, involucre and flowers details, C. Long stiles and stigmas details (Photographs by A. Moreira-Muñoz). 
Hallazgo de Nardophyllum genistoides: MuÑOz-SCHICK, M. ET AL.

Clave para las especies de Chile

1. Hojas verdes en ambas caras, con ancho de $2-8 \mathrm{~mm}$

N. genistoides

1'. Hojas blanquecinas, al menos en una cara, con ancho menor de $2 \mathrm{~mm}$

2. Tallos con 5 costillas, a veces poco notorias por lo densamente tomentosas.

N. bryoides

2'. Tallos con 8 costillas notorias

3. Hojas concolores, blanquecinas, de 9-18 mm de largo, tallos erectos; vilano con pelos plumosos en el ápice

N. lanatum

3'. Hojas discolores, de (3-) 8-16 mm de largo, tallos intrincado-ramosos y ramas a veces terminadas en puntas espinosas; vilano con pelos no plumosos en el ápice....

N. chiliotrichioides

MATERIAL ADICIONAL

Región Metropolitana, Reserva Nacional Río Clarillo, Puerta de la Guanaca, I-2010, J. Solervicens s.n. (SGO 159016); Reserva Nacional Río Clarillo, Puerta de la Guanaca, $33^{\circ}$ 47'18,9" S, 70²5'55,6" W, aprox. 1.600 m, 3-II-2010, A. Moreira, V. Morales \& J. Solervicens 1288 (SGO 159017).

\section{OBSERVACIONES SOBRE SU CONSERVACIÓN}

Por lo escaso de la especie se presentará a CONAMA para ser incluida en la lista de especies con problemas de conservación. La planta estaría relativamente protegida ya que se encuentra en la Reserva Nacional Río Clarillo, aunque su baja abundancia le otorga el carácter de muy rara. Esta nueva localidad más al sur es un aporte al conocimiento de su distribución geográfica, ya que la localidad original es Las Arañas, ubicada más al norte en la misma Región Metropolitana.

Ahora se presenta el desafío de cultivar la especie ex situ y estudiar su biología tanto en terreno como bajo cultivo; igualmente buscar nuevos individuos en cajones aledaños, y en comunidades semejantes.

\section{AGRADECIMIENTOS}

Proyecto Fondecyt Iniciación 11085016 (2008). Jaime Solervicens, entomólogo y naturalista, conocedor de la Reserva, ha puesto su ojo observador al servicio del proyecto. Carlos Peña, administrador de la Reserva, el jefe de guardaparques Rogelio Moreira y el asistente Segundo Navia han aportado el apoyo logístico en las expediciones. Agradecemos a Mauricio Bonifacino (Uruguay) por transmitirnos su entusiasmo por este grupo tan atractivo de plantas. A Roberto Kiesling por su esclarecimiento en dudas nomenclaturales. A uno de los revisores anónimos por los importantes datos bibliográficos aportados.

\section{BIBLIOGRAFÍA}

Bonifacino, J.M. 2008. Reinstatement of Ocyroe (Compositae: Astereae). Brittonia 60(3): 205-212.

Bonifacino, J.M. 2009. Taxonomic Revision of the Chiliotrichum Group sensu stricto (Compositae: Asteraceae). Smithsonian Contributions to Botany 92: 1-119.

Cabrera, A.L. 1954. Las especies del género Nardophyllum. Notas Museo La Plata, Botánica 17: 55-66.

Gray, A. 1873. Nardophyllum genistoides. Proceedings American Academy of Arts and Sciences 8: 636

PhiLIPPI, R.A. [1856] 1858. Plantarum novarum chilense. Linnaea 28, Parte sexta: 641-767.

Stafleu, F.A. \& R.S.Cowan. 1985. Taxonomic literature. Second edition. Utrecht vol. 5: Sal-Ste, 1066 pp.

Teillier, S., G. Aldunate, P. Riedemann \& H. Niemeyer. 2005. Flora de la Reserva Nacional Río Clarillo, Guía de identificación de especies. Universidad de Chile-CONAF, 367 pp.

Recibido: 23.03 .09

Aceptado: 19.04 .10 